\title{
Innovación y desarrollo social: ¿es posible la construcción de una relación estratégica?
}

\author{
Peña Cedillo, Jesús* \\ Petit, Elsa Emilia**
}

\section{Resumen}

Se discute el papel complejo y contradictorio de la innovación al ser abordada su relación con el desarrollo social. Se realizó un estudio cualitativo crítico-documental, caracterizándose los fundamentos de la innovación a partir del contraste y análisis de autores clásicos como Smith, Marx, Schumpeter, Veblen y contemporáneos v.gr. Rostow, Freeman, Nelson, Sundbo; así como su relación con las dinámicas socio-productivas y de desarrollo, atendiendo, por ejemplo, a las tesis de North, Bourdieu, Elster, Castells, Stiglitz. La innovacion es un impetuoso fenómeno generador de riqueza, pero solo para sectores sociales acotados; justo por ello es también el principal diferenciador al interior del sistema socio-económico predominante. Se concluye sobre la urgente necesidad de nuevos enfoques capaces de cuestionar la prevalencia de las lógicas marcadas por los mercados y el capital, superando las perspectivas neo-clásicas y evolucionistas hoy en día preponderantes. Es menester refundar los procesos sociales de producción y reproducción del conocimiento y sus aplicaciones, sobre nuevas bases económicas, epistemológicas y axiológicas, aportadas por actores sociales y económicos emergentes.

Palabras clave: Innovación, desarrollo social, economía neoclásica, economía evolucionista, transformación social.

\section{Recibido: 10-01-12. Aceptado: 23-06-13}

* Doctor en Ciencias Políticas. Magíster Scientiarum en Planificación del Desarrollo. Profesor Titular de la Universidad Simón Bolívar (USB-Caracas), adscrito al Departamento de Ciencias Económicas y Administrativas. Investigador PPI Nivel III. Miembro del Laboratorio Estudios de la Innovación y el Desarrollo, e-mail: jpenac@usb.ve

** Doctora en Ciencias Humanas. Magister Scientiarum en Planificación y Gerencia de Ciencia y Tecnología. Profesora Titular de la Universidad del Zulia (LUZ-Maracaibo), adscrita a la Unidad Académica Estudios del Desarrollo, Departamento de Ciencias Humanas, Facultad Experimental de Ciencias. Investigadora PEI Nivel B. Miembro del Laboratorio Estudios de la Innovación y el Desarrollo, e-mail: elsapetitluzve@gmail.com 


\title{
Innovation and Social Development: Is it Possible to Construct a Strategic Relationship?
}

\begin{abstract}
The complex and contradictory role of innovation is discussed, approaching its relationship to social development. A qualitative, critical documentary study was carried out, characterizing the foundations of innovation based on the contrast and analysis of classic authors such as Smith, Marx, Schumpeter, Veblen and contemporaries v.gr. Rostow, Freeman, Nelson and Sundbo. Relation of the foundations of innovation to social-productive and development dynamics was studied according to the theses of North, Bourdieu, Elster, Castells and Stiglitz. Innovation is an impetuous phenomenon that generates wealth, but only for limited social sectors. Precisely because of this, it is also the principle differentiator within the predominant socio-economic system. Conclusions are that there is an urgent need for new approaches able to question the prevalence of logic marked by markets and capital, overcoming today's preponderant neo-classical and evolutionist perspectives. It is necessary to re-found the social processes of production and re-production of knowledge and their applications on new economic, epistemological and axiological bases contributed by emerging social and economic actors.
\end{abstract}

Keywords: Innovation, social development, neoclassical economy, evolutionist economy, social transformation.

\section{Introducción}

Se presentan en este trabajo algunos elementos teóricos e históricos vinculados con la innovación y su importancia, no solo para el crecimiento económi$\mathrm{co}$, sino principalmente para el desarrollo social. Mediante un análisis crítico-documental se estudian los fundamentos teóricos de la innovación, revisando y contrastando en profundidad a los principales autores que aportan elementos sustantivos para el abordaje del tema, tanto clásicos como contemporáneos (entre otros, Schumpeter, 1934 [1911], 1939; Robinson, 1933; Veblen, 1969 [1898]; Mandel, 1975; Marx, 1977 [1867-1894]; Rosenberg, 1979; Rostow, 1978; Freeman et al, 1982; Dosi, 1982; Pavitt, 1984; North, 1984, 1995; Bourdieu, 1990; Mokyr, 1990; Elster, 1991-1992; Nelson,
1995; Castells, 1996-1998; Sundbo, 1998; Stiglitz, 2002).

Las relaciones entre innovación y crecimiento económico han sido ampliamente documentadas (entre muchos: Pavitt, 1984; Nelson, 1995; Bryant, 2001), habiéndose establecido con claridad su importante papel en la generación de riqueza material; pero no existe tal evidencia cuando se trata de entroncarla con fenómenos más complejos como los involucrados con el desarrollo económico y social, en particular en sociedades no industrializadas de la periferia o la semi-periferia mundial, como es el caso de la América Latina.

Se trata, por tanto, de una temática de extraordinaria importancia, ya que afecta los fundamentos que deben guiar las políticas públicas en diversas áreas (desde la científico-tecnológica hasta la 
económica), llegando su alcance hasta la manera como deben ser pensadas e implementadas las propuestas estratégicas de desarrollo, no solo en el nivel nacional, sino en términos de toda la región latinoamericana.

En tal sentido, los recientes procesos de transformación socioeconómica que están en curso en la mayoría de los países de la región, han establecido como un asunto crucial alcanzar el desarrollo priorizando las políticas sociales (Ffrench Davis, 2004; Kliksberg, 2006; Coraggio, 2010); cambiando el énfasis antes prevaleciente en las políticas económicas aperturistas (en los años noventa incluso se llegó a afirmar que tales políticas sociales no eran necesarias, sino que derivaban automáticamente de las económicas) (Petkoff, 1997; Borón, 2005).

Este notable cambio de énfasis ha producido resultados considerados más que satisfactorios por la mayor parte de los analistas y los organismos internacionales (v.gr. BID, 2006; UNESCO, 2010; CEPAL, 2011; FAO, 2012); pero aún subsisten espacios de discusión de mucha importancia a la hora de conectar ambos tipos de políticas.

Uno de esos espacios difusos es el que sostiene que la promoción de la innovación, tal como ha sido pensada e implantada en los países desarrollados, puede y debe ser adoptada (copiada) en Latinoamérica con el fin de dirigir esos fenómenos hacia propósitos de desarrollo económico y social integral; con ello aparentemente se lograría mantener el privilegio de lo social, sin sacrificar las perspectivas de crecimiento económico.

Justamente, la inadecuación e imposibilidad de una aproximación como la señalada (sustentada en la reproducción en Latinoamérica de las experiencias en torno a la innovación provenientes de Europa y Norteamérica) es el eje de discusión central de este estudio.

En tal sentido, se intenta aclarar en profundidad que el abordaje de las políticas de innovación a partir de un planteamiento mimético, originalmente dirigido a perfeccionar el sistema capitalista internacional, entraría en conflicto directo con la estrategia de desarrollo que procura la prevalencia del componente social.

Junto con la discusión crítica antes señalada, se avanza en el planteamiento de las nuevas perspectivas que conecten la innovación con el desarrollo social de una manera distinta; esto es: se propone el establecimiento de una relación estratégica (lo que implica políticas públicas, cultura, redistribución de poderes, etc.) en el desenvolvimiento de ambos fenómenos.

Una investigación tal solo puede abordarse a partir de un paradigma epistemológico crítico y trans-disciplinario; y así se ha hecho, apelando a las herramientas propias del análisis socio-político, pero aplicadas a la complejidad social (que obliga, por tanto, al análisis conjunto de lo económico, lo sociológico, lo político, lo tecnológico y lo cultural).

Todo ello se ha concretado en un estudio cualitativo crítico-documental en donde se caracterizaron, por un lado, los fundamentos de la innovación (asentados principalmente en la teoría económica), gracias al contraste y análisis de autores tanto clásicos (entre otros, Smith, Marx, Schumpeter, Veblen) como más contemporáneos (v.gr. Rostow, Freeman, Nelson, Sundbo, Dagnino); y por 
otro lado se discutieron las relaciones de la innovación con las dinámicas socioproductivas y sus impactos en el desarrollo social (atendiendo, por ejemplo, a las tesis de North, Bourdieu, Elster, Varsavsky, Castells, Stiglitz).

Se abordan -sin pretensión de ser exhaustivos- algunos elementos esenciales; en particular las diferencias presentes entre las aproximaciones neo-clásicas ortodoxas versus los paradigmas evolucionistas e institucionalistas que se han disputado el campo durante el siglo pasado. De igual manera, se discuten los límites que tienen estas aproximaciones a la innovación, los cuales evitan que desde ellas se pueda realizar un abordaje adecuado de la temática del desarrollo social. Se sostiene, como contrapartida, que para tratar con posibilidades reales de incidencia las relaciones entre innovación y desarrollo, se requieren posturas analíticas y políticas que rompan definitivamente con las lógicas del mercado y del capital.

\section{Trabajo, innovación, bienestar y riqueza}

En la tarea de discernir lo fundamental de los fenómenos bajo estudio, es ilustrativo y pertinente comenzar con Adam Smith (1997 [1759]). Durante la segunda mitad del siglo XVIII, el autor de La Riqueza de las Naciones dirigía la Cátedra de Ética en la Universidad de Edimburgo. Ejerciendo esas funciones, sostenía que no era verdad que los seres humanos poseyeran la virtud de la benevolencia o de la caridad, y que cada ser humano era en esencia egoísta y pensaba en sus propios intereses. A pesar de ello, aclaraba Smith, de la suma de estos egoísmos individualistas surgía la riqueza de las naciones, produciéndose un extraordinario bien para el conjunto de los seres humanos.

En efecto, Smith lograba con una tesis novedosa la resolución de una aporía ética clásica:

"Si cada uno es egoísta y lucha por sus propios intereses [...] luchando cada uno solamente por el egoísmo en el mercado que es la gran mediación, entonces la mano de Dios toma el egoísmo de cada uno y lo transforma en el trabajo no coordinado por nadie, no intencional, obra del puro mercado, pero éste aún regido por la mano providente de Dios, y de allí sale la riqueza nacional" (Smith, 1997 [1759]: 19).

Esta expresión, en su contexto histórico original, debe entenderse como expresamente religiosa (Smith afirma que sin su Dios cristiano -que se manifestaría a través de los mercados, en su gran innovación conceptual- no habría solución a la aporía). Por supuesto, con posterioridad y en los ámbitos considerados como científicos, se prefirió desnudar un poco la expresión, privilegiándose otra frase del mismo Smith: la acción extraordinaria la ejerce la mano invisible del mercado, lugar de la mediación económica que transforma el egoísmo en riqueza (Smith, 1997 [1776]:402).

Pero Smith también dejó otra herencia -usualmente olvidada- para entender el fenómeno que importa en esta discusión: Adam Smith nunca dejo de considerar el trabajo humano como fuente de todo valor en el proceso económico. En pleno siglo XXI esos dos elementos cen- 
trales siguen presentes en cualquiera de los debates modernos: ¿Qué papel se debe asignar a los mercados en el desarrollo social y económico? ¿Qué importancia tiene o no el trabajo humano en la generación de la riqueza económica y en el progreso social? En adelante se hará patente que tales consideraciones son de gran interés para dilucidar la principal interrogante que está siendo tratada: ¿es teóricamente posible establecer/construir una relación estratégica entre innovación y desarrollo social?

\section{El desarrollo social como problema}

Las dinámicas de crecimiento y desarrollo han sido ampliamente discutidas en la literatura científica (sean las sociales, en general; o las económicas y políticas, en específico); reconociéndose una gran variedad de matices (o francas contradicciones) entre las diversas posturas (entre muchos, véanse: Rostow, 1961; Gunder Frank, 1967; Freyssinet, 1976; Marx, 1977 [1867-1894]; Samuelson y Nordhaus, 2010). Se deben señalar entonces los fundamentos que guían este estudio en torno a una de sus categorías clave: el desarrollo social.

En un sentido muy básico, el desarrollo social es la garantía de un nivel adecuado de calidad de vida para todas las personas. Se trata de un proceso que conduce al mejoramiento de las condiciones de vida de toda (o la gran mayoría de) la población de una sociedad en múltiples ámbitos (salud, educación, nutrición, vivienda, vulnerabilidad, seguridad social, empleo, salarios, participación) (Midgley, 1995).
Se trata del cambio positivo en las relaciones de individuos, grupos e instituciones en una sociedad, en procura (simultánea, concurrente, inseparable) de desarrollo económico y humano. No hay duda de que se trata de un proceso complejo, mediado por determinaciones culturales, económicas y políticas; siendo su concreción el bienestar social; no se confunda, por tanto, con crecimiento económico.

Una de las teorías más difundidas que explica que tales procesos se hayan dado relativamente acabados en los países más industrializados, se sustenta en planteamientos como los de T.H. Marshall (actualizados por Bottomore) sobre la ciudadanía y la democracia, según los cuales existe una tendencia inevitable hacia la igualdad en virtud de que ella acompaña la evolución del concepto de ciudadano (con los derechos sociales sustantivos que éste englobaría) (Marshall y Bottomore, 1998). Se trata, sin duda, de tesis estrechamente enraizadas en las teorías modernizadoras, ahora difundidas con tintes neoclásicos, que culminaron con el planteamiento (luego rechazado incluso por su propio autor) acerca del 'fin de la historia' (Fukuyama, 1989, 1995).

De acuerdo con estas teorías, el desarrollo social es precedido en los países industrializados por la formación de un Estado nacional, por el desarrollo industrial autónomo y por el desarrollo económico en general. En términos de ciudadanía, nos encontraríamos con el surgimiento de la ciudadanía civil en el siglo XVIII, de la ciudadanía política en el siglo XIX y de la ciudadanía social en el siglo XX (Marshall y Bottomore, 1998). Solo en 
este último caso el desarrollo social tendría cabida y posibilidades de ser realizado. Eso sí, la tesis se cuida de señalar que tal desarrollo que iguala en bienestar social, no tiene que ir acompañado de ninguna igualdad económica (lo que importaría sería el 'enriquecimiento general' de toda la ciudadanía).

Incluso pensada la sociedad desde esas teorías, en las condiciones de subdesarrollo de la América Latina la tarea de construcción paulatina y secuencial de formación del Estado nacional, desarrollo industrial, democracia y bienestar social, ha tenido que ser afrontada simultáneamente (cuando ello se ha intentado); apelándose a muy distintos consensos sociales y a muy distintas herramientas económicas y políticas. Las consecuencias de este proceso tan distinto al supuesto para otras latitudes, han demostrado que son impracticables los mismos criterios teóricos y prácticos para la transformación económica y social de nuestras sociedades; lección que a duras penas parece que recién ha sido aprendida a inicios del siglo XXI (luego del más reciente y desastroso intento mimético representado por la noche neoliberal de finales del siglo XX) (Ffrench Davies, 2004; Borón, 2005; Kliksberg, 2006).

En efecto, la 'búsqueda' del desarrollo social por la vía secuencial marshalliana en lo socio-político y neoclásica en lo económico, que en apariencia fue la que condujo al desarrollo en los países industrializados, se plasmó en los años noventa en los países de Latinoamérica en costos representados por una profunda crisis distributiva y de bienestar; el mayor empobrecimiento de la población en términos de aumento de la desigualdad y la pobreza; la disminución notoria de la participación de los salarios en el ingreso nacional; la precariedad del acceso a los bienes públicos; la violencia política; los bajos coeficientes de inversión privada; los efectos negativos combinados en la calidad de vida de la población, en particular de mujeres, niños y jóvenes; la erosión de la calidad potencial de la mano de obra (por la desnutrición y la crisis de la educación), etc., etc.

En fin, al hacer desaparecer las bases materiales para acceder al bienestar $y$, al mismo tiempo, convivir con el ejercicio mediatizado de la democracia; la creencia neoliberal condujo a una inestabilidad social, económica y política tan generalizada, que terminaron por llevar al colapso a prácticamente todos los sistemas políticos de la región.

La experiencia latinoamericana ha dejado en evidencia que el funcionamiento libre de los mercados jamás resolverá por si solo el tema de la desigualdad y del subdesarrollo social. Por el contrario, ella sostiene la crítica a la teoría neoclásica en el sentido de que la distribución del bienestar está dada exógenamente a los procesos puramente económicos; por tanto, el equilibrio distributivo no puede ser un resultado simple del mercado, sino que es un tema que pertenece al campo de la política.

Es en ese marco de dinámica social no lineal, contradictoria y conflictiva, no necesariamente 'progresiva'; que se proponen las políticas públicas reales en economía, en derechos democráticos, en derechos sociales. Allí también es que se da el debate sobre cómo la innovación contribuye o no a tales procesos de desarrollo social. 


\section{Un cambio de época en la economía y en la sociedad}

Se requiere abordar el fenómeno del cambio tecnológico para poder examinar si la innovación puede ser efectivamente una herramienta con incidencia sustantiva en el desarrollo social (más allá del mero crecimiento económico).

El mundo se encuentra desde hace algunas décadas en medio de una transformación histórica. Como cualquiera de las grandes mutaciones que ha sufrido la humanidad, es multidimensional, expresándose en esferas tales como la tecnología, la economía, la política, la geopolítica, la cultura y hasta en la psicología de los individuos y las agrupaciones sociales. Con ella han estado viniendo aparejadas alteraciones extraordinarias en el desarrollo social y en la vida de la gente. Aunque no todos se están viendo afectados de la misma manera (Castells, 1996-1998).

El cambio de época está signado por la complejidad de las lógicas económicas que han estado emergiendo, con un creciente papel de los procesos de generación y difusión de conocimiento con características complejas y altamente interactivas gracias a la fertilización cruzada de ideas entre sectores, actividades y agentes de distinta naturaleza.

Desde una perspectiva normativa, planificar y sustentar el desarrollo en un contexto tal es sin duda una tarea mucho más difícil. Un eje crítico para reforzar el análisis de la situación lo constituye la consideración del tema de la auto-organización o interacción coordinada de los numerosos agentes que operan en los sistemas productivos complejos, en pro- cura de que los instrumentos que promuevan la creatividad y la innovación garanticen el pleno desarrollo humano y la construcción de sociedades más equitativas y justas.

Pero el nuevo orden social que se está desplegando pareciera tener otras características. Una aproximación que resuma lo esencial puede ser construida con base en algunas de las visiones diversas más difundidas (por ejemplo, Castells, 1996-1998; Touraine, 1998; Nweihed, 1999; Stiglitz, 2002). A partir de ellas se podría señalar que en la actualidad se presencia el efecto combinado de tres procesos que surgieron hacia los años setenta del siglo pasado, los cuales, juntos, están produciendo una nueva sociedad. Estos fenómenos son, a saber (Castells, 19961998):

- La revolución de las tecnologías de la información,

- La crisis económica del estatismo, fuese cual fuese su signo, y

- El florecimiento de nuevos movimientos sociales, como el ambientalista, el feminista y una gran variedad de localismos comunitarios.

Existen lazos estrechos entre estos elementos, pero es imposible detenerse en ello en este trabajo. Simplemente es importante conocer que, en su conjunto, tales procesos están generando una nueva estructuración económica (que profundiza el sistema capitalista mundial), nuevas estructuras sociales (que subsumen su lógica a las propias de la economía liberal) y nuevas manifestaciones de la cultura (en las que se prioriza la virtualidad). Se trata de procesos contradictorios que igualmente forjan estructuras 
(económicas, sociales, culturales y políticas) que se oponen a la nueva estructuración dominante.

El impulso más importante hacia este nuevo tipo de organización social puede fecharse en los años 90 , cuando la parte más opulenta del planeta procedió a organizarse alrededor de las redes de computadoras telecomunicadas, las cuales se constituyeron en el corazón de los sistemas de información y de los procesos de comunicación de la nueva era. Debido a ello, el campo entero de la actividad humana pasó a depender del poder de la información, en una secuencia recurrente de innovaciones tecnológicas que acelera su paso día a día (Elster, 1991, 1992; Lander, 1994; Castells, 1996-1998; Hughes, 1997; Touraine, 1998).

Si bien la difusión de estas tecnologías ha sido muy desigual, creándose incluso zonas que podrían denominarse como de apartheid tecnológico en varias partes del mundo, esto parece ser parte de la lógica misma de esta manera de construir el nuevo orden social, y no un problema de mala implementación del diseño, superable en cuanto se caiga en cuenta de ello.

Debe quedar claro que las tecnologías de la información y la comunicación no son en sí mismas la causa de estas transformaciones; aunque sin ellas ninguno de los cambios mencionados sería posible. De allí la necesidad de abordar este fenómeno desde una perspectiva más amplia, no centrándose exclusivamente en el caso específico de las tecnologías de la información, sino en el fenómeno más general que las atrapa: el cambio tecnológico y la innovación.

\section{El papel de los paradigmas tecnológicos}

En efecto, el final del siglo XX ha permitido presenciar el despliegue de una transición tecnológica global, con serias consecuencias para las oportunidades de desarrollo. Pero tal fenómeno no es completamente inédito; la historia de la humanidad en general, y de las sociedades capitalistas en particular, ha atravesado por períodos de intensa transformación tecnológica en varias oportunidades. En este sentido, es indispensable hacer referencia a los estudios relacionados con las ondas largas de Kondratiev o van Gelderen, para colocar en su contexto esas macro-transformaciones (véanse, por ejemplo, los análisis que desde perspectivas muy diversas se encuentran en Mandel, 1975; Rostow, 1978; Freeman et al., 1982; Maddison, 1998; y Sundbo, 1998).

Para entender la significación de estos fenómenos debe comprenderse el concepto de sistema tecnológico. Los sistemas tecnológicos son constelaciones (Keirstead, 1948) de innovaciones interconectadas técnica y económicamente, que afectan a múltiples ramas del sistema económico. Sobre esta temática son de interés los aportes de Hughes (1997), para quien los sistemas tecnológicos constituyen conjuntos complejos y desordenados de artefactos físicos, organizacionales, empresas, bancos de inversión, órganos legislativos y elementos humanos -inventores, científicos, ingenieros, gerentes, financistas, trabajadores y usuarios de los 'artefactos'-, todos contribuyentes a la innovación tecnológica. 
El cambio en un sistema tecnológico se reproduce como un encadenamiento de sucesivas innovaciones radicales interrelacionadas en lo que aparenta ser una trayectoria natural global. Una vez se reconoce y entiende la lógica del sistema tecnológico prevaleciente, pareciera hacerse posible entender y aprovechar la sucesión creciente de nuevos productos y procesos que podría deparar (Pérez, 1986).

Debe destacarse que el conjunto de innovaciones interrelacionadas no se reduce solamente a las innovaciones puramente técnicas, sino que éstas se entremezclan con innovaciones sociales, organizativas y gerenciales (Freeman et al., 1982). Al ampliarse la visión y reconocerse la existencia de innovaciones radicales con una notable capacidad para transformar todo el aparato productivo, se está ante una constelación de sistemas tecnológicos con una dinámica común. Su difusión a lo largo y ancho del sistema productivo termina por englobar la casi totalidad de la economía.

Estas revoluciones conducen a profundos cambios estructurales y de ellas existen diversos ejemplos: la era del ferrocarril a mediados del siglo XIX, la electricidad en la Belle Epoque, el motor de combustión interna, la línea de ensamblaje, entre otros, para no hablar del microchip o lo que ya está representando la ingeniería genética. Cada uno de esos casos resume el impacto que determinados sistemas tecnológicos han tenido sobre la economía mundial y la manera de vivir.

Diversos razonamientos han sido adelantados para explicar por qué se dan esos encadenamientos de innovación tan poderosos. Algunos autores como por ejemplo, Pérez (1985) han señalado que cada revolución tecnológica se basa en una modificación radical y duradera en la dinámica de costos relativos del conjunto de todos los posibles insumos del proceso productivo, estableciendo que algunos tenderán a la baja y otros al alza por largos períodos de tiempo. Surgiría así un 'tipo ideal' de organización productiva capaz de hacer las previsiones correctas para lograr las combinaciones más eficientes durante un período largo. Esta cadena de acontecimientos terminaría orientando las decisiones de inversión y de innovación tecnológica, lo cual se presentaría en el panorama estratégico como el despliegue de un paradigma tecno-económico.

El término paradigma le calza perfectamente a esta descripción, en virtud de que se ha tratado de una creencia, una percepción, que al ser compartida por los colectivos decisionales más importantes, ha llegado a convertirse en el 'sentido común' de los ingenieros, gerentes e inversionistas, para el logro de niveles superiores de eficiencia.

Obsérvese que esta explicación se opone a las tesis tradicionales de que las decisiones de inversión se toman exclusivamente en función de los costos relativos del trabajo y del capital. En ese sentido acompaña a Freeman et al. (1982), quienes señalan que es iluso suponer que una decisión de largo alcance se tome con base en pequeñas variaciones en el costo relativo de los factores y no en función de un cambio significativo, usualmente sostenible durante un largo plazo. Aun así, la explicación de Pérez se asemeja demasiado a las aproximaciones de signo racionalista centradas en la búsqueda de óptimos de eficiencia en el largo 
plazo (por ello, luego se expondrán y discutirán críticamente las tesis evolutivas y de aprendizaje que más recientemente intentan explicar el fenómeno, rompiendo definitivamente con estas visiones todavía clásicas).

Las interpretaciones de esta naturaleza han llegado al punto de señalar que son los procesos de despliegue de nuevos paradigmas tecno-económicos los que explican desde la conducta oportunista individual de los agentes económicos aislados, hasta el comportamiento de las ondas largas de Kondratiev; esto es, el fenómeno de la innovación debería ser considerado crucial para poder explicar tanto los fenómenos de competencia directa entre agentes económicos, como los fenómenos de transformación estructural del sistema económico mundial. Aquí se corre el peligro de entregarle todos los argumentos a lo que se denomina determinismo tecnológico.

El determinismo tecnológico supone que la lógica del cambio estrictamente técnico arrastra, conduce y moldea el resto de los fenómenos sociales. Un derivado de esta tesis es que debe esperarse un progreso socio-económico sostenido, inevitable, neutral y con tendencia a generalizarse. Para el caso de los países subdesarrollados (Rodríguez, 1993; Sampedro, 1996), el determinismo tecnológico implicaba el seguimiento de la vía trazada por los países occidentales industrializados, sin mayores consideraciones sobre las lógicas que les son particulares como organizaciones sociales diferentes (Lander, 1994; Ramos, 1999).

Es por ello que, para superar ese determinismo, se hace necesario recalcar que los paradigmas tecno-económi- cos a lo sumo lo que podrían estar estableciendo es un amplio campo de posibilidades, dentro del cual los actores sociales están en capacidad de experimentar arreglos diversos para competir o cooperar. En ese sentido, las opciones tecno-económicas no derivan en una receta o solución única, a pesar de que insistentemente así se haya tratado de 'vender' la historia del desarrollo de las sociedades (véase al respecto las muy diversas rutas seguidas por los Estados Unidos, Francia, Japón o China, para mencionar sólo algunos ejemplos, en sus respectivos y muy diferentes caminos hacia niveles superiores de calidad de vida, puestas en evidencia por los estudios de North, 1984; Mokyr, 1990; Maddison, 1998; Landes, 1999).

De igual manera, debe señalarse que las sociedades no son sólo resultado de la transformación tecnológica y económica. En el planeta permanentemente surgen vigorosos movimientos sociales que propugnan maneras de hacer sociedad que no se subordinan a imperativos tecno-económicos, sino que por el contrario hasta aparecen para enfrentarse a ellos (verbi gratia, los movimientos que provocaron la detención súbita que sufrió en su momento el desarrollo y uso de la energía atómica, la oposición de importantes sectores a lo que se ha denominado 'la sociedad de consumo' o el despliegue vigoroso de las corrientes que impulsan el desarrollo sustentable).

Por supuesto, las transformaciones estructurales planteadas por estos cambios paradigmáticos, no ocurren sin conflictos. Frente al despliegue de las nuevas opciones, el desarreglo inicial que se produce en las instituciones socia- 
les y el marco socio-económico prevaleciente, obliga a que los cambios vayan más allá del ámbito puramente técnico y económico. Usualmente todo el entramado socio-institucional debe ser reconstruido, en un proceso conflictivo que no es de corto plazo. La capacidad de los grupos humanos para resolver estos períodos turbulentos y la correlación de fuerzas socio-políticas, los proyectos y concepciones de sociedad que resultan dominantes, dictan el derrotero que seguirá el paradigma asimilado.

En ese sentido, la dinámica predominante del proceso estructurador que hoy se despliega ha conducido a resultados poco halagadores en la mayor parte del mundo: aumento de la desigualdad, mayor polarización social, incremento de la pobreza y la miseria. El despliegue del paradigma tecnológico informacional ha estado creando o remarcando, según sea el caso, una aguda línea divisoria entre pueblos y localidades; con un pequeño conjunto que es considerado valioso, en tanto se deja de lado una inmensa mayoría que es considerada como prescindible.

En la base de esta situación se encuentra el hecho de que el paradigma tecnológico ha sido cabalgado por un conjunto de fuerzas sociales que han privilegiado la globalización financiera y han avanzado en las transformaciones de forma selectiva, incluyendo y excluyendo a segmentos completos de economías y sociedades de la participación y el disfrute de las redes de información, de la riqueza y del poder que caracterizan el nuevo sistema que se despliega.

No se está en presencia de un gran proceso especulativo, sino ante una lógica de poder: la del dominio de los merca- dos financieros y los grupos sociales que los aprovechan (Touraine, 1998; Stiglitz, 2002) sobre todas las demás oportunidades de inversión. La movilidad del capital y la interconexión de la producción crean las condiciones para que la inversión se traslade instantáneamente y a bajo costo por el mundo entero, atraída por zonas de costos económicos inferiores. Las nuevas tecnologías de la información conducen este torbellino global de acumulación de riqueza y difusión de pobreza, y no es de extrañar que en torno a su despliegue se hayan estado formando los agujeros negros del capitalismo informacional actual (Castells, 1996-1998).

Es importante destacar que los períodos de transición tecnológica proveen también a las sociedades relativamente atrasadas una opción para la modificación de la productividad de su sistema económico, con su correspondiente impacto tanto en la estructuración de los diversos grupos o actores sociales, como en la posición relativa que pueda tener un país en la distribución de la riqueza mundial.

En circunstancias de turbulencia se abren espacios para la búsqueda de nuevas maneras de aprovechar el despliegue del nuevo paradigma, intentando evitar las variantes perversas que hoy en día predominan. Por supuesto, el despliegue de esas nuevas posibilidades, se encuentra mediado por las restricciones que imponen las diferentes historias y las muy distintas realidades socio-económicas de partida que encara cada sociedad. De igual manera, hay lógicas tecnológicas asociadas muy fuertemente a determinados modelos de vida (por ejemplo, el consumista o el depredador del ambiente) que pueden ser contradictorias con los nuevos 
proyectos socio-políticos que se impulsan en Latinoamérica. De allí que la linealidad de cualquier 'oportunidad' tecnológica debe ser puesta profundamente en duda.

El conjunto de fenómenos macrosociales y macro-tecnológicos que se han abordado, forman parte del sustrato explicativo del fenómeno de la innovación. En particular, debe atenderse a que sólo a través de su comprensión y adecuado manejo puede pretenderse conjugar los fenómenos globalizadores versus los que mantienen un estricto marco nacional; así como los que podrían apuntar a un desarrollo creciente de la productividad con calidad de vida versus los que demuestran que la innovación es un fenómeno por su naturaleza diferenciador y excluyente, y que por tanto requiere ser conducido con detalle, si se desean minimizar los costos sociales que con él pueden estar asociados.

\section{Ortodoxia económica e innovación}

Deben explorarse con detenimiento algunos detalles específicos sobre la innovación. Innovar es producir y poner en práctica con incidencia social y/o económica nuevo conocimiento. Esta definición diferencia el fenómeno de la innovación de otro con el cual tiende a confundirse: la invención. Esta última, a fin de cuentas, es la realización de alguna idea científica, teoría, concepto o artefacto, que en sí mismo puede no producir ningún efecto socio-económico importante. A continuación se destacan algunos elementos característicos de importancia:
- En primer lugar, el hecho notorio de que los procesos de innovación no son en general lineales. La innovación se nutre de múltiples fuentes que la inician, impulsan o transforman; fuentes que abarcan un amplísimo rango que va desde descubrimientos científicos fundamentales hasta las señales del mercado.

- En segundo término, es un hecho comprobado que las tecnologías tienden a mantenerse cambiando incrementalmente mientras se difunden; esto implica que tecnologías ya establecidas pueden rejuvenecerse, dominando así la competencia frente a las nuevas. Es decir: no necesariamente lo nuevo tiene éxito sobre tecnologías de más vieja data.

- En tercer lugar, más que aparecer aisladamente, las nuevas tecnologías tienden a emerger en paquetes que incluyen múltiples elementos complementarios a la innovación principal (experticias, inversiones sustanciales en infraestructura, nuevas relaciones, entre otros). Usualmente se requieren innovaciones paralelas y nuevas competencias, cuya aparición o no, puede acelerar o ralentizar el proceso innovativo.

- Un cuarto fenómeno puede producirse cuando en un sector o industria un comportamiento excesivamente auto-reforzante (lo que en cierto sentido podría considerarse una sobre-adaptación o el estancamiento en un óptimo local) limita las innovaciones radicales, conduciendo al bloqueo de todo el proceso innovativo.

Agréguese a esta ya compleja situación el hecho de que no todas las in- 
dustrias siguen comportamientos equivalentes al desarrollar estos procesos. Sólo a manera de ejemplo, la taxonomía adelantada por Pavitt (1984) según la cual es posible identificar, entre otras, industrias dominadas por los proveedores, industrias que son intensivas en escala, industrias intensivas en información e industrias que se basan en la ciencia. Al tiempo que cada uno de estos grupos tiene sus características propias, también son comunes los casos de empresas y subsectores que entrecruzan varias de estas categorías. A toda esta mezcla, agréguense las categorizaciones diferentes que deben hacerse en el área de los servicios y en otros sectores no manufactureros.

Un elemento central que merece consideración detallada es la naturaleza social del proceso de innovación, el cual, más que fundamentarse en el tratamiento de los asuntos tecnológicos materiales, está fuertemente asociado con las habilidades individuales y colectivas para aprender, estar alertas ante las oportunidades y desarrollar competencias para poner en práctica las ideas (Bryant, 2001). Efectivamente, el tema de la innovación está estrechamente vinculado con el manejo del conocimiento.

No deja de ser extraño que hablar del conocimiento en economía aparezca en el momento actual como algo totalmente novedoso, porque en algún sentido toda la teoría económica ha tratado siempre sobre información y conocimiento. Sirva de ejemplo Adam Smith, quien ya en su época había planteado como un asunto crucial estudiar no solo cómo los agentes individuales tomaban decisiones con base en la información provista por los mercados, sino también cómo se re- solvían los problemas de coordinación entre los agentes (Smith, 1997 [1776]).

Ciertamente, una distinción crucial entre las diferentes teorías económicas surge de los postulados que ellas utilizan acerca de cómo los agentes conocen y acerca de en qué grado aprenden algo a partir de su quehacer. Esta distinción separa claramente, por ejemplo, a los economistas neoclásicos de los de la escuela austriaca; con los primeros considerando como el caso de referencia aquél en el cual los agentes están completamente informados (Friedman, 1956, 1970), en tanto los últimos enfatizan la ignorancia como el punto de partida para el aprendizaje (Schumpeter, 1934: [1911], 1939). También el elemento conocimiento siempre ha separado a quienes asumen la híper-racionalidad (incluyendo las expectativas racionales) como el caso típico (Sargent, 1976; Begg, 1982), en contraposición con aquéllos que asumen como punto de partida la racionalidad limitada (Robinson, 1933; Chamberlin, 1946).

La economía clásica se enfoca sobre los problemas de la colocación de recursos en un contexto de equilibrio general. La perspectiva básica es una en donde los agentes que tienen unas preferencias dadas y una cantidad de información específica, incluyendo un stock dado de conocimiento tecnológico público compartido, se comprometen en realizar elecciones racionales. Sobre la base de este conjunto de presunciones, se llega a conclusiones normativas acerca de cómo debería ser organizado y conducido el sistema económico. Esta perspectiva teórica deja poco espacio a la incertidumbre genuina, ya que la escasez está en el corazón del análisis. Por supuesto, puede ser 
un esquema útil para la comprensión de asuntos parciales o de corto plazo, pero es menos adecuado para el análisis del desarrollo económico, y menos aún para estudiar el desarrollo social.

En este orden de ideas, no se dice nada nuevo cuando se afirma que el neoclasicismo económico está en su elemento cuando se enfrenta con medios estáticos. Es por ello que extender dichas tesis al problema dinámico de la innovación es problemático (Elster, 1992; Bryant, 2001). La ortodoxia neoclásica tiende a explicar el cambio tecnológico como simplemente otro caso de maximización bajo limitaciones, centrándose en conceptos idealizados acerca de los mercados e introduciendo elementos como las fallas de mercado que obstaculizan que éstos alcancen el estado de equilibrio.

Una dificultad fundamental para el desarrollo adecuado de esta perspectiva surge del hecho de que, si bien la innovación en cualquier momento dado se encuentra limitada por lo que es técnicamente posible (entre muchas otras cosas), estas limitaciones no pueden entrar en la explicación de la innovación a no ser que sean conocidas por anticipado por el innovador. Por tanto, aunque se suponga que el empresario innovador maximiza ganancias, no se le imputa el conocimiento del conjunto factible de innovaciones que permitiría explicar su conducta con basamento en el modelo estándar de la racionalidad paramétrica.

En definitiva, no es razonable analizar los procesos de aprendizaje y de innovación sin considerar la incertidumbre fundamental del fenómeno. No tomarla en cuenta llevaría a la contradictoria conclusión de que los agentes conocen por adelantado todas las cosas que deberían conocer (o acceden al conocimiento sin ninguna barrera) y que los innovadores conocen anticipadamente todos los posibles resultados de los procesos de innovación.

Por lo demás, los estudios sobre la innovación han revelado un fenómeno complejo que envuelve múltiples actores e influencias, con actividades que se organizan y nutren dentro de un sistema dinámico de múltiples niveles (desde el nivel global hasta diferentes categorías de niveles locales, comunitarios y empresariales) y, por tanto, no existe una 'mejor' manera de gerenciar esos procesos de innovación, ni una conducta particular que maximizar.

Se requiere una perspectiva que reconozca como natural la existencia de las asimetrías y la falta de información, las limitaciones del comportamiento racional ante las restricciones que las instituciones imponen, lo crucial de los comportamientos adaptativos y de búsqueda activa del aprendizaje, la importancia del conocimiento (y su creciente expresión como una forma del capital) y la coexistencia de fenómenos de cooperación en estrecho contacto con los de competencia. Estas características calzan difícilmente dentro de los esquemas económicos neoclásicos.

\section{La propuesta no ortodoxa: instituciones y evolución}

La teoría de la evolución por variación casual y por selección natural es inmensamente atractiva ya que es a la vez simple y poderosa, de allí que haya sido utilizada en innumerables ocasiones en las ciencias sociales como metáfora ex- 
plicativa útil. En ese sentido, la noción general de que el avance tecnológico procede a través de un proceso evolutivo ha sido desarrollada independientemente por estudiosos del tema en variedad de disciplinas (en sociología, historia de la tecnología, economía, artes militares, gerencia empresarial, entre otros).

Ahora bien, de entrada debe atenderse a que en el terreno de la economía las tesis evolucionistas sólo comparten un parentesco débil con la evolución biológica y de ninguna manera se asumen todas sus derivaciones. Así, por ejemplo, la evolución económica necesariamente tiene que incluir la intencionalidad humana como uno de sus elementos, lo que afecta seriamente la suposición de que toda la variabilidad es casual y obliga a hacer consideraciones explícitas acerca de las características materiales y socioculturales de estos fenómenos.

El asunto central en cualquier teoría evolucionista no es el ser sino el devenir, resolver el problema de por qué el mundo cambia y la manera en que lo hace, incluyendo a qué velocidad y en qué dirección. Es aquí donde la perspectiva evolucionista en economía resulta sumamente útil en virtud de que sugiere que aquellos elementos que para los neoclásicos son asimetrías e imperfecciones, realmente constituyen el combustible que impulsa la innovación (Nelson, 1995).

El desarrollo del pensamiento evolutivo en el campo económico ha permitido identificar muchas ideas que le dan sentido al término innovación, tanto desde una perspectiva social, como técnica y organizacional; enlazando el fenómeno innovador con temas cruciales como el crecimiento económico, el cambio estructural y los procesos competitivos. Esas mismas ideas enfatizan el papel que juegan los procesos de interacción y coordinación en la economía, bien sea a través de los mercados o los sistemas de innovación, y subrayan que se está en presencia de una dinámica de cambio turbulenta que no necesariamente conduce hacia un 'óptimo'. Las imperfecciones, brechas, ineficiencias y ausencia de lazos dentro de los sistemas, son características usuales que están en permanente evolución, no existiendo realmente un estado de equilibrio privilegiado, ni atractores de estabilidad que produzcan ciertos procesos convergentes. Para el pensamiento económico estándar estás afirmaciones constituyen herejía y no es de extrañar que las posiciones evolucionistas sean en economía una contra-cultura anti-ortodoxia.

Los sistemas económicos son abiertos y necesariamente dinámicos. Si adicionalmente se consideran sistemas evolutivos, deben cambiar de acuerdo a procesos de características muy peculiares: el desarrollo de variedad y la selección de la misma. Se trata de fenómenos por naturaleza dinámicos que se despliegan en múltiples niveles y presentan retroalimentación positiva (reforzante), con todo lo que esto implica en términos de lo que se denomina path dependency o historia propiamente dicha.

Desde un plano ligeramente diferente, aparece aquí el tema crucial de la auto-organización como una dimensión de la evolución en economía. El comportamiento evolutivo auto-organizado puede considerarse como un patrón de comportamiento que surge de la interacción (típicamente localizada) entre los componentes de un sistema. Ese patrón no ne- 
cesariamente se repite en cada individuo, los cuales pueden comportarse de manera diferenciada. Es en el agregado que emergen las propiedades de las interacciones que lo componen.

Desde la perspectiva auto-organizacional los sistemas basados en conocimiento son adicionalmente auto-catalíticos: el conocimiento se alimenta a sí mismo generando más conocimiento en formas usualmente impredecibles. De allí que el atributo crucial de los agentes económicos no sea (no pueda ser) la búsqueda racional de la eficiencia, sino más bien la construcción imaginativa e incierta de mundos económicos futuros alternativos (Loasby, 1999). En un mundo económico evolutivo, es definitivamente más productivo inquirir acerca de cómo sobreponerse a la ignorancia, que postular una perfecta previsión como la base del comportamiento económico.

En este punto se hace necesario destacar la importancia de las instituciones. Atiéndase a que se concibe por instituciones los mecanismos que ponen en vigencia reglas (Elster, 1991). Más aún, desde una perspectiva plenamente institucionalista, las instituciones son en sí mismas el conjunto de reglas, los procedimientos de aceptación y cumplimiento de las mismas, y las normas éticas y morales que se diseñan para restringir el comportamiento de los individuos (North, 1984).

El evolucionismo y el institucionalismo en economía comparten una premisa conductual básica en el sentido de que procuran entender la acción y la interacción humana como el resultado de hábitos para pensar y actuar compartidos. Ahora bien, aquí se interpreta la definición de hábitos a la manera de Bourdieu (1990): un producto de la historia que produce acciones individuales y colectivas de acuerdo con los esquemas generados por las prácticas del pasado; esquemas de percepción, pensamiento y acción que garantizan la 'rectitud' de las prácticas y su constancia en el tiempo, incluso más confiablemente que las reglas formales o las normas explícitas. Hábitos que, en definitiva ( $y$ esto es trascendente para marcar una crucial distancia con el institucionalismo neoclásico), se encuentran sustentados en la vida real que los seres humanos construyen a partir de sus prácticas de trabajo, comunicación y cooperación.

A partir de lo dicho, es claro que quedan en un segundo plano los procesos de maximización del bienestar individualista como lo característico del comportamiento económico de los seres humanos. Tanto en el campo del evolucionismo como del institucionalismo, se presenta el patrón de acción humano como un fenómeno que se modifica con el tiempo gracias a procesos de aprendizaje individual y colectivo.

Si bien es cierto que desde la perspectiva evolucionista todo fluye, resulta ser que esto sucede en ámbitos institucionales específicos que en mayor o menor medida facilitan la interacción y la coordinación (y los mercados deben reconocerse sólo como uno de esos arreglos institucionales). Es así como la historia y el sustrato material de los procesos interactivos, los costos de transacción, el juego de poderes socio-económicos precedentes, las visiones del mundo, la incertidumbre y la confianza, aparecen como problemas centrales para los agentes, con igual estatus que el poseído por los precios y costos de los mercados neoclá- 
sicos entre otros, North (1984), (1995); Elster (1991); Fukuyama, (1998).

Igualmente, a la luz del planteamiento institucionalista cabría diferenciar dos tipos de evoluciones: la northiana, que trata de la evolución institucional en el marco de una economía nacional o supranacional; y la schumpeteriana, que se refiere a las instituciones al interior de las organizaciones; reconociéndose las interrelaciones inevitables entre los dos niveles.

En el campo macro aparecen como elementos institucionales relevantes las tradiciones de cooperación, los derechos de propiedad, las leyes antimonopolio, los proyectos ideológicos nacionales, la formación general de recursos humanos, la provisión de infraestructura; elementos todos que constriñen los ensayos innovadores que en el nivel meso y micro serán realizados. Por su lado, en estos últimos niveles las empresas y otros tipos de organizaciones ponen de relieve la modificación de hábitos, las capacidades de influenciar legisladores, los valores que se comparten, entre otros, haciendo énfasis en el desarrollo de estrategias de interacción micro que demuestran tener capacidad para orientar la evolución de la innovación en un nivel más general.

Pero no se trata de una incidencia unilateral de las instituciones sobre el cambio técnico. Aquéllas también están fuertemente condicionadas por la manera como las nuevas tecnologías son desarrolladas, aceptadas y absorbidas dentro del sistema socio-económico, así como por el camino tecnológico que efectivamente se transita (Dosi, 1982; Elster, 1992).

Llevado el argumento a su extremo, ya Veblen en 1898 -y antes que él pero con otras terminologías, Marx (1867) y Smith (1776)- señalaban que las mismas instituciones son sujetos de selección, así que no pueden ser asumidas como dadas exógenamente. Más aún, las instituciones pueden llegar a ser modificadas e incluso creadas por las unidades que se supone ellas deben seleccionar: jen buena medida, la innovación trata con la anticipación y creación de las futuras condiciones de selección!

En definitiva, el evolucionismo y el institucionalismo en economía coinciden en reconocer un conjunto de fuerzas, actores e influencias que usualmente son excluidas del análisis económico estándar; $y$, en particular, la tecnología y las instituciones deben ser consideradas como co-evolucionando en un fenómeno que deviene en la principal fuerza subyacente al proceso de crecimiento y desarrollo económico.

Pero lo cierto es que estas posiciones no pueden negar su origen: se trata de visiones que intentan entender en profundidad el funcionamiento del sistema capitalista globalizado, con el fin de mejorar los esquemas de aprovechamiento de la innovación, sin poner en cuestionamiento las consecuencias perversas que tienen estos fenómenos para grandes cuerpos sociales (usualmente ubicados en espacios ajenos a los países desarrollados).

\section{Más allá de la teoría: ¿ha producido la innovación desarrollo social?}

Decía Nietzsche (1973 [1886]: 23) que "es destino de todo mito irse deslizando a rastras, poco a poco, en la estrechez de una presunta realidad, para ser tratado por un tiempo posterior cualquie- 
ra como un hecho ocurrido una vez, con pretensiones históricas". Y sabido es que resulta vergonzoso para el hombre actual admitir que su accionar está orientado por móviles de orden mítico. Sin embargo, el mito no es ajeno a las relaciones sociales supuestamente avanzadas: se instala silenciosamente en la sociedad occidental como respuesta a frustraciones, desencantos e incertidumbres. Gracias a ellos se asientan proyectos de mundo, se reduce la ansiedad y se hace manejable el temor.

Evidentemente, se está ampliando el sentido usual que se le asigna al término, según el cual: "los mitos son historias sagradas elaboradas por el hombre primitivo para explicar su realidad, el origen del mundo, animales, plantas y el hombre, transmitidas de generación en generación, en donde los protagonistas son seres divinos" (Escamilla, [2003: s/p]). Se enfatiza más bien que "el mito cuenta cómo, gracias a las hazañas de seres sobrenaturales, una realidad ha venido a la existencia, convirtiéndose en el modelo ejemplar de todas las actividades humanas significativas [...] El mito no habla de lo que ha sucedido realmente" (Eliade, [2003: s/p]).

Pero el mundo occidental no ha estado exento de producir y convivir con sus propios mitos. Así como la Modernidad desarrolló el mito de la 'Edad de Oro': la supuesta existencia de una sociedad $\sin$ conflictos ni tensiones sociales, futuro de orden y progreso al que llegaría toda la humanidad a través de la racionalidad científica; así como en gran parte del siglo XX se creyó que el Estado de Bienestar promovería el desarrollo, viviéndose la ilusión de que se llegaría a una sociedad armónica y equitativa; así igualmente se depositaba hacia finales de siglo toda la confianza en la acción suprema del Mercado, ya que éste - se decía- busca siempre optimizar las ganancias y los beneficios. Así, mediante un juego de libre intercambio, se produciría el bienestar de todos, librándose al individuo del hambre y de la pobreza. Smith dixit.

En el ámbito que aquí atañe, también se ha convivido con un mito equivalente. ¿No es acaso claro que también la innovación ha sido considerada en ciertos discursos como una palanca fundamental para lograr el crecimiento económico que las sociedades requieren para su progreso y para, con ello, alcanzar el mejor bienestar de todos?

Ciertamente, se trata de la reiteración de lo planteado en épocas pasadas cuando se hablaba de la ciencia como panacea. Y así como el mito no era fundado en este último caso, existen suficientes evidencias de que tampoco lo es en materia de innovación.

Lo cierto es que es creciente el descreimiento de grandes grupos sociales frente al estilo de desarrollo dominante a nivel global, ampliamente anclado en el fenómeno de la innovación y en la explotación económica del desarrollo tecnológico en ambientes dominados por las lógicas de los mercados; estilo de desarrollo que se ha revelado ecológicamente predatorio, socialmente perverso y políticamente injusto.

Junto con ello, el paro estructural, la degradación de la calidad de las relaciones de trabajo de las mayorías y la exclusión socio-económica generalizada, son el trasfondo de ese discurso apologético acerca de la innovación y el desarro- 
Ilo tecnológico, aun dominante en la economía y la política actual.

Estudios realizados en diversas oportunidades durante la última década confirman que la innovación, dejada a su propia dinámica, es un fenómeno profundamente diferenciador en el seno del cuerpo social, y a partir del dejar hacer, dejar pasar, se logra -inexorablementetanto la producción de extraordinarias riquezas para pocos sustentadas en los beneficios propios del éxito innovador; como la generación de grandes segmentos sociales, mayoritarios ellos, inmersos en la pobreza y la miseria, en convivencia yuxtapuesta y cada vez más inestable con esas grandes acumulaciones de riqueza (Fagerberg et al., 1997; World Bank, 2000).

En efecto, esa visión optimista del papel de la ciencia, la tecnología y la innovación en relación con el desarrollo de las sociedades, no deja de ser-strictu sensuun mito contemporáneo. $Y$ las visiones teóricas acerca de la innovación, como se ha repasado, no rompen de ninguna manera con esa pretensión, con ese mito.

Pareciera necesario entonces someter a esas visiones meramente instrumentales, falsas e inconsistentes, además de socialmente injustas, a un proceso de re-aprendizaje que haga aflorar de nuevo la crítica.

Se trata de la necesidad de construir una alternativa de acción para las sociedades en el ámbito de la innovación, que logre encauzarlas dentro de una lógica que permita construir desarrollo social aprovechando el altísimo potencial para la generación de riqueza que indudablemente posee el cambio técnico. Se trata de lograr una efectiva relación estratégica entre innovación y desarrollo que se sustente en tesis rigurosas y sustentables.

Precisar algunos elementos es fundamental. En primer lugar, si bien el ámbito preferido en el que se habla de innovación es el sector usualmente llamado productivo (término en el que, por lo general, sólo se engloba -erróneamente- la actividad industrial, manufacturera y comercial), el concepto también se refiere a otras actividades sociales, vale decir: nuevas formas organizacionales, nuevos tipos de asociación, e incluso la distribución no comercial de nuevos productos o descubrimientos demostradamente útiles. Este uso del término implica entonces incorporarle el concepto de cambio social (North, 1984; Elster, 1992).

En segundo lugar, la innovación debe entenderse como una fuerza económica parcialmente controlable, a pesar de todas las complejidades antes destacadas. La innovación se produce y se difunde en función de un conjunto de factores sociales, económicos y políticos que pueden ser amoldados a distintos escenarios de prioridades económicas y sociales.

En efecto, no ha perdido sentido la sugerencia de Nathan Rosenberg (1979) de que es necesario abrir 'la caja negra' del cambio técnico para comprender sus mecanismos constitutivos y para reconsiderar la relación entre la ciencia, la tecnología y la sociedad, todo ello no según una lógica lineal y unidireccional, sino sistémica, evolutiva y crítica.

Tómese como ejemplo el análisis que hace Christopher Freeman y su escuela (Freeman et al., 1982), promotores de acercamientos sistémicos y evolutivos (aunque poco críticos) al proceso innovador. Ellos establecen que las consecuen- 
cias iniciales del cambio técnico son, sin duda alguna, violentas y destructoras; en particular por sus efectos en el empleo, en la obsolescencia de las profesiones y de los capitales tradicionales; pero, por otro lado, consideran demostrado que se generan nuevas funciones profesionales y financieras, así como nuevos productos y servicios. La escuela de Freeman concluye que su análisis del balance del empleo es positivo, siempre y cuando se esté hablando de high-tech.

Es claro que tal no es la situación en el conjunto del mundo, sometido, en el juego político y económico mundial, a la generalización interesada de esas visiones estrechas y solo parcialmente válidas en los contextos de las sociedades desarrolladas.

Se hace imprescindible recapitular: la innovación no es sólo la aplicación de los resultados de investigación y desarrollo de alto nivel, sino que también es el resultado del estado de la situación en las relaciones sociales, de las capacidades emprendedoras existentes, de las pautas estratégicas en curso, del juego de poder prevaleciente y de las capacidades para tomar decisiones en los niveles micro y macro en el seno de la sociedad.

Vinculado con lo anterior, recuérdese que la relación tecnología-empleo se funda en un paradigma cultural del siglo pasado: el de la eficiencia productiva basada en el ahorro de tiempo y de esfuerzo; por lo tanto, es intrínseco a su contenido el hecho de que la tecnología actual presente rendimientos decrecientes en términos del empleo global. Es evidente entonces la paradoja tecnológica que contrapone a quien trabaja demasiado con quien no puede hacerlo, a los centros de excelencia con las áreas de pobreza creciente.
De hecho, las políticas de la innovación más populares son sólo aquellas que favorecen el desplazamiento hacia adelante de la frontera tecnológica; es decir, aquellas que promueven la innovación radical. Se obvian, entonces, las políticas de carácter integral, sustentadas sobre las características ciertas del territorio, únicas que permitirían al mayor número de actores sociales y a los distintos Estados y a las diversas regiones de un mismo Estado, correr a la misma velocidad o de un modo menos deficiente.

Aquí es pertinente recalcar la concepción de desarrollo social que se encuentra en juego: un proceso que, en el transcurso del tiempo, conduce al mejoramiento de las condiciones de vida de toda la población en diferentes ámbitos: salud, educación, nutrición, vivienda, vulnerabilidad, seguridad social, empleo, salarios. Implica también la reducción de la pobreza y la desigualdad en el ingreso (Midgley, 1995). Por tanto, muy lejos se está de equiparar desarrollo social con crecimiento económico; tampoco se trata de su más reciente mimetización con algunas formas de capital (capital social, capital humano, entre otros) (Putnam, 2000; Sala-i-Martin, 2000).

Por ello, afrontar el carácter disfuncional y el poder disruptivo intrínseco a la tecnología en el marco del capitalismo, parece pasar por el cuestionamiento de las lógicas de acumulación dominantes en estas sociedades, si es que efectivamente se quieren superar las desigualdades que produce la innovación; particularmente en contextos en donde las diferencias sociales y económicas de partida son muy profundas entre sus distintos estratos. De lo contrario, en estos espacios 
ampliamente desestructurados se está propiciando que los fenómenos de cambio técnico sometidos a las lógicas de los mercados y el capital sigan siendo un impedimento y no una herramienta para el logro de objetivos de desarrollo social.

¿Será posible, entonces, replantear la apertura de la caja negra del proceso innovador en una clave que no sea la típica lectura mimetizadora de lo que proviene de las economías industrializadas? Más todavía, ¿será posible plantearse el tema de la sus relaciones con el desarrollo social en un marco que deje de privilegiar, por fin, el mito propagado por Smith acerca de la prevalencia de los mercados y el capital?

En esta dirección vale la pena citar a Varsavsky (1974), quien en sus estudios sobre los estilos tecnológicos y los estilos de desarrollo, señaló que los latinoamericanos al plantearse sus propios objetivos productivos, encuentran que la tecnología y la innovación no tienen respuesta para muchísimos de los problemas prácticos que esos objetivos obligan a resolver. Postula entonces que se adopte una decidida 'actitud creativa y transformadora' para construir sus propios estilos de desarrollo y sus propios modelos innovativos.

Décadas después, Dagnino (1996) igualmente concluye que la innovación, para que se convierta en práctica efectiva y eficaz de cualquier nación de Latinoamérica, obliga a promover una cultura tecnológica apropiada a las necesidades y características de los pueblos de la región. Ello lo asocia directamente con la superación de la lógica capitalista que impide a los sectores productivos nacionales desarrollar sus propias capacidades innovativas; solo con esa ruptura será posible que ellas se orienten a contribuir al bienestar social de sus conciudadanos, privilegiando en esa nueva lógica el trabajo y no el capital.

Dagnino deja en claro que se trata de un desafío a la vez científico, político e ideológico: un reto para la construcción de la perspectiva social que debe entretejer los conceptos de ciencia, tecnología e innovación. En fin, se identifica como principio básico para la construcción del bienestar social latinoamericano, la resolución adecuada y estratégica (en múltiples dimensiones y en función del largo plazo) de las relaciones entre innovación y desarrollo social.

Tal perspectiva hoy en día se integra a la concepción de desarrollo que emerge en el continente: un desarrollo integral que abarca como ejes centrales concurrentes la preservación de los ecosistemas, el impulso al crecimiento económico desde y hacia adentro, y la promoción del desarrollo humano (Peña Cedillo, 2006; Urdaneta y Petit, 2008; Carrero y Petit, 2011).

Mas expresamente, la construcción de cohesión social, el desarrollo organizacional innovador, la promoción de la colaboración y la reciprocidad, la cocreación, el trabajo en red, y el empoderamiento popular; surgen como los fundamentos cohesivos de ambos fenómenos.

Todo lo dicho hace perfectamente transparente que la interfaz compleja entre innovación y desarrollo social es altamente ideológica y política. Y es ilusorio no enfrentarse a ello. Más aún, en ese cuestionamiento se insiste que el problema no se reduce a la creación de interacciones distintas entre ambas esferas, 
sino que está planteado cuestionar profundamente la lógica misma de los procesos de innovación que se auspician, porque desde allí se afecta la posibilidad de vinculación entre los dos fenómenos. ¿Será posible, entonces, replantear la apertura de la caja negra en una clave que no sea la típica lectura mimetizadora de lo que proviene de las economías desarrolladas? Más todavía, ¿Será posible plantearse el tema de la innovación y sus relaciones con el desarrollo social en un marco que deje de privilegiar, por fin, el mito propagado por Smith acerca de la prevalencia de los mercados y el capital?

\section{A manera de conclusión}

A la luz de lo presentado, todo estudioso de la innovación y el desarrollo debe caer en cuenta de que las explicaciones ortodoxas que intentan asumirse a sí mismas como la única opción de explicación científica del fenómeno innovador, pecan de los errores generales que el neo-clasicismo en su conjunto sigue cometiendo en economía: un notable desprecio por los fenómenos asociativos, por los cambios debidos a aprendizajes y por la aleatoriedad inherente a la interacción colectiva de los humanos; amén de las ya conocidas deficiencias intrínsecas a los planteamientos que suponen la existencia de los mercados perfectos.

En este marco, los planteamientos evolucionistas e institucionalistas surgen como opciones más adecuadas para aproximarnos al estudio de los fenómenos que implican innovación, particularmente cuando la perspectiva es indagar cómo pueden ser conducidos hacia el logro de propósitos que van más allá del crecimiento económico en el espacio de un capitalismo mucho más complejo y turbulento que el concebido desde el neoclasicismo.

Pero las tesis evolucionistas han surgido para explicar el capitalismo en contextos desarrollados y sus conclusiones en torno a la dinámica de provisión de empleo o de derrame de beneficios, solo parecen ser adecuadas en aquellos segmentos más avanzados en la asimilación del cambio técnico. En tal sentido y en los contextos propios de los países subdesarrollados, estas tesis han pasado a convertirse en una nueva ortodoxia que tiende a ser asimilada acríticamente.

Habida cuenta de que los más trascendentales resultados del análisis conducen a considerar a la innovacion como un impetuoso fenómeno generador de riquezas para sectores sociales acotados; y justo por ello actúa como el principal diferenciador de los grupos humanos al interior del sistema socio-económico predominante; no es plausible plantear como un automatismo que la innovación conduzca al desarrollo social; muy por el contrario, las características dominantes del fenómeno innovador están produciendo crecientes desequilibrios, en particular en aquellas sociedades ya de por sí desestructuradas y desiguales.

En concordancia con lo anterior, lo que se hace evidente es la urgencia de nuevos enfoques sobre la innovación que con claridad sean capaces de cuestionar la prevalencia de las lógicas marcadas por los mercados y el capital, y que superen las perspectivas neo-clásicas y evolucionistas hoy en día preponderantes. No parece posible extender las fronteras de impacto social de la in- 
novación respetando y reproduciendo las reglas del capitalismo globalizante que hoy se despliega, porque las restricciones derivadas de la prevalencia tanto de los mecanismos de mercado dominados por los consumidores más solventes, como de la apropiación privada de los beneficios económicos que de la innovación se derivan, hacen que los movimientos a favor del desarrollo social sean solo marginales y reactivos.

Es menester, por tanto, desarrollar una perspectiva mucho más radical: lo que está planteado es refundar los procesos sociales de producción y reproducción del conocimiento y sus aplicaciones; y ello debe hacerse necesariamente sobre nuevas bases económicas, epistemológicas y axiológicas aportadas por actores sociales y económicos emergentes y con modelos de acumulación radicalmente distintos.

No es el propósito de este texto el desarrollo pleno de esa visión alternativa, pero es claro que ella no podrá sustentarse en el predominio de la lógica de los mercados y el capital en el funcionamiento económico de la sociedad; muy por el contrario, cualquier nueva aproximación que quiera dar cuenta de una relación cierta entre innovación y desarrollo social, deberá dar una respuesta muy diferente a una cuestión básica: quiénes tienen el poder de disponer del conocimiento en cuanto a su uso y aprovechamiento (esto es, quiénes son los sujetos sociales que orientan el esfuerzo científico, tecnológico y de innovación, qué problemas se resuelven, qué valores se producen y entre quiénes se distribuyen). Lo que sí es posible adelantar son los que, a juicio del argumento expuesto, serían algunos de los ejes fundacionales a priorizar, anclados en el hecho de que son el trabajo y los actores sociales a ellos asociados los que en definitiva producen la riqueza social. Se considera urgente auspiciar el desarrollo de procesos de aprendizaje social, el predominio de mecanismos de apropiación social del conocimiento, y la refundación (económica, institucional, valorativa y participativa) de los procesos sociales de producción y reproducción del conocimiento.

La construcción de una relación estratégica fructífera entre innovación y desarrollo solo será viable y efectiva para grandes segmentos sociales, en la medida que se consideren y prioricen esos elementos. A fin de cuentas, lo que se propone es desarrollar procesos socio-económicos sustentados en innovación, pero no sujetos ni al imperio de los mercados orientados por los consumidores opulentos, ni a la apropiación privada de los beneficios sociales y económicos de la innovación. Se recuperará así, joh paradoja!, el pensamiento económico de Adam Smith (luego desarrollado por Ricardo y por Marx), cuando señalaba que en el proceso económico el trabajo humano es la fuente de todo valor.

\section{Referencias Bibliográficas}

Begg, David (1982). The rational expectations revolution in macroeconomic: theories and evidence. Oxford, Philip Allen.

Bourdieu, Pierre (1990). The logic of practice. Cambridge, UK, Polity Press.

Bryant, Kevin (2001). Promoting innovation: an overview of the application of evolutio- 
nary economics and systems approaches to policy issues. En: Foster, John y Stanley Metcalfe (eds.) (2001). Frontiers of evolutionary economics: competition, self-organization and innovation policy. Cheltenham, Edward Elgar.

Carrero, Wilmer y Elsa Petit (2011). Aspectos del desarrollo social para la innovación desde la perspectiva de la corriente del pensamiento creativo $\mathrm{y}$ transformador latinoamericano. Omnia, Vol. 17, N. 1, pp. 52-66.

Castells, Manuel (1996-1998). The Information Age: Economy, Society and Culture. 3 Vol. Cambridge, Mass, Blackwell.

Chamberlin, Edward (1946). The theory of monopolistic competition. Cambridge, Mass, Harvard University Press.

Dagnino, Renato (1996). Innovación y Desarrollo Social: Un Desafío Latinoamericano. Campinas, UNICAMP.

Dosi, Giovanni (1982). Technological paradigms and technological trajectories. Research Policy, Vol. 11, pp. 147162.

Eliade, Mircea [2003]. Ensayo de una definición del mito. [Documento electrónico] [http://www.unimag.edu.co/antropologia/ensayo_de_una_definicion_del_mito.htm] [Sin paginación] [Consultado el 25-10-2008].

Elster, Jon (1991). Tuercas y tornillos: una introducción a los conceptos básicos de las ciencias sociales. BarceIona, Gedisa.

Elster, Jon (1992). El cambio tecnológico: investigaciones sobre la racionalidad y la transformación social. Barcelona, Gedisa.

Escamilla, Soledad [2003]. Sentido común. [Documento electrónico] [http://www. monografias.com/trabajos15/sentido-comun/sentido-comun.shtml] [Sin paginación] [Consultado el 25-102008].

Fagerberg, Jan; Verspagen, Bart y Caniëls Marjolein (1997). Technology, growth and unemployment across European regions. Regional Studies, Vol. 31(5).

Freeman, Christopher; Clark, John y Luc Soete (1982). Unemployment and technical innovation: a study of Long Waves in economic development. London, Pinter.

Friedman, Milton (1956). The quantity theory of money. Chicago, University of Chicago Press.

Friedman, Milton (1970). Positive Economics. Chicago, University of Chicago Press.

Fukuyama, Francis (1998). La confianza (Trust). Barcelona, Grupo Zeta.

Hughes, Thomas (1997). The evolution of large technological systems. En: Bijker, W.; Hughes, T. y T. Pinch (eds.) (1997). The social construction of technological systems. Cambridge, Mass, MIT Press.

Keirstead, Burton (1948). The theory of economic change. Toronto, Macmillan.

Lander, Edgardo (1994). La ciencia y la tecnología como asuntos políticos: límites de la democracia en la sociedad tecnológica. Caracas, Nueva Sociedad.

Landes, David (1999). The wealth and poverty of nations. New York, Norton.

Loasby, Brian (1999). Knowledge, institutions and evolution in economics. London, Routledge.

Maddison, Angus (1998). Historia del desarrollo capitalista: sus fuerzas dinámicas. Barcelona, Ariel.

Mandel, Ernest (1975). Late capitalism. London, New Left Books.

Marx, Karl (1977 [1867-1894]). El capital: crítica de la economía política. México, Fondo de Cultura Económica. 
Midgley, James (1995). Social Development: The Developmental Perspective in Social Welfare. London, Sage.

Mokyr, Joel (1990). Lever of riches: technological creativity and economic progress. New York, Oxford University Press.

Nelson, Richard (1995). Recent evolutionary theorizing about economic change. Journal of Economic Literature. March, pp. 48-90.

Nietzsche, Friedrich (1973 [1886]). El nacimiento de la tragedia. Madrid, Alianza.

North, Douglass (1984). Estructura y cambio en la historia económica. Madrid, Alianza.

North, Douglass (1995). Instituciones, cambio institucional y desempeño económico. México, Fondo de Cultura Económica.

Nweihed, Kaldone (1999). Globalización: dos rostros y una máscara. Caracas, Instituto de Altos Estudios de América Latina.

Pavitt, Keith (1984). Sectoral patterns of technical change: towards a taxonomy and a theory. Research Policy, Vol. 13, pp. 343-373.

Peña-Cedillo, Jesús (2006). Redes de Innovación Productiva: un análisis económico y político en la perspectiva del Socialismo del Siglo XXI. Caracas, MCT.

Pérez, Carlota (1985). Microelectronics, long Waves and Structural Change: new perspectives for developing countries. World Development, Vol. 13(3), pp. 441-463.

Pérez, Carlota (1986). Las nuevas tecnologías: una visión de conjunto. Santiago de Chile, PREALC.

Putnam, Robert (2000). Bowling Alone: The Collapse and Revival of American Community. New York, Simon \& Schuster.
Ramos, Mauricio (1999). Modern technology and technological determinism: the Empire strikes again. Bulletin of Science, Technology \& Society, Vol. 19(5), pp. 403-410.

Robinson, Joan (1933). The economics of imperfect competition. London, Macmillan.

Rodríguez, Octavio (1993). La Teoría Del Subdesarrollo de la Cepal. Madrid, Siglo XXI.

Rosenberg, Nathan (1979). Economía del cambio tecnológico. México, Fondo de Cultura Económica.

Rostow, Walt (1978). The World economy. London, Macmillan.

Sala-i-Martin, Xavier (2000). Apuntes de crecimiento económico. Barcelona, Antoni Bosch.

Sampedro, José Luis (1996). Conciencia del subdesarrollo veinticinco años después. Barcelona, Taurus.

Sargent, Thomas (1976). Rational expectations and the term structure of interest rates. Journal of Political Economy, Vol. 84(2), pp. 207-237.

Schumpeter, Joseph (1934 [1911]). The theory of economic development. Cambridge, Mass, Harvard University Press.

Schumpeter, Joseph (1939). Business Cycles: a theoretical, historical and statistical analysis of the capitalist process. New York, McGraw-Hill.

Smith, Adam (1997 [1759]). Teoría de los sentimientos morales. México, Fondo de Cultura Económica.

Smith, Adam (1997 [1776]). Investigación sobre la naturaleza y causas de la riqueza de las naciones. México, Fondo de Cultura Económica.

Stiglitz, Joseph (2002). El malestar en la globalización. Madrid, Taurus.

Sundbo, Jon (1998). The theory of innovation: entrepreneurs, technology 
Innovación y desarrollo social: ¿es posible la construcción de una relación estratégica? Peña Cedillo, Jesús y Petit, Elsa Emilia

and strategy. Cheltenham, UK; Northampton, Mass; Edward Elgar.

Touraine, Alain (1998). ¿Podremos vivir juntos? México, Fondo de Cultura Económica.

Urdaneta, Emilio y Elsa Petit (2008). Política de Innovación para el Desarrollo Integrado. Ponencia presentada en el 27 Congreso Internacional CIRIEC. Sevilla, España.

Varsavsky, Oscar (1974). Estilos Tecnológicos: Propuesta para la Selección de Tecnologías bajo Racionalidad Socialista. Caracas, MCTI.

Veblen, Thorstein (1969 [1898]). Why is Economics not an evolutionary science? En: Veblen on Marx, Race, Science and Economics, New York, Capricorn.

World Bank (2000). Entering the 21st century: World Development Report 1999/2000. New York, Oxford University Press. 